\title{
Occurrence, distribution, and potential affecting factors of organophosphate flame retardants in sewage sludge of wastewater treatment plants in Henan Province, Central China
}

\author{
Long Pang a, b, *, Yiting Yuan ${ }^{\text {a }}$, Han He ${ }^{\text {a }}$, Kang Liang ${ }^{c}$, Hongzhong Zhang ${ }^{\text {a, b }}$, \\ Jihong Zhao ${ }^{\text {b, } * *}$ \\ ${ }^{a}$ Department of Material and Chemical Engineering, Zhengzhou University of Light Industry, Zhengzhou, Henan 450001, People's Republic of China \\ ${ }^{\mathrm{b}}$ Collaborative Innovation Center of Environmental Pollution Control and Ecological Restoration, Zhengzhou, Henan 450001, People's Republic of China \\ c State Key Laboratory of Environmental Chemistry and Ecotoxicology, Research Center for Eco-Environmental Science, Chinese Academy of Sciences, Beijing \\ 100085, People's Republic of China
}

\section{H I G H L I G H T S}

- The levels of organophosphate esters (OPEs) in Henan province were lower than those of South China.

- Chlorinated OPEs were predominant in sewage sludge.

- Triphenyl phosphate (TPhP) level in sewage sludge depends on wastewater characteristics.

- The levels of OPEs in sewage sludge were independent of the total organic carbon (TOC) and solid retention time.

\section{A R T I C L E I N F O}

\section{Article history:}

Received 12 October 2015

Received in revised form

2 January 2016

Accepted 24 February 2016

Available online 12 March 2016

Handling Editor: Caroline Gaus

Keywords:

Organophosphate flame retardants

Wastewater treatment plant

Sewage sludge

\begin{abstract}
A B S T R A C T
Organophosphate esters (OPEs) are widely used as flame retardants. In this study, the occurrence and distribution of six OPEs were investigated in sewage sludge from 24 wastewater treatment plants (WWTPs) in 18 cities of Henan province, Central China. The results indicated that all target OPEs were detected in the sludge samples with the detection rate of $95.8 \%$, except tris(dichloropropyl)phosphate (TDCP). The total concentration of the six OPEs ranged from 38.6 to $508 \mu \mathrm{g} \mathrm{kg}^{-1}$. Tris(2-chloroethyl) phosphate (TCEP), tris(2-butoxyethyl)phosphate (TBEP), and tris(2-chloroiso-propyl)phosphate (TCPP) were found to be predominant, with concentrations ranging from 2.50 to $203,1.60$ to 383 , and 6.70 $-161 \mu \mathrm{g} \mathrm{kg}^{-1}$, respectively. The potential factors affecting OPE levels in sewage sludge, such as wastewater source, sludge characteristics, operational conditions, treatment techniques, and total organic carbon (TOC) of sludge in WWTPs were investigated. The results indicated that the total concentration of OPEs in sewage sludge has no significant relationship with the individual parameters $(p>0.05)$. However, significant correlations were found between triphenyl phosphate (TPhP) level and treatment capacity $(R=0.484, p<0.05)$, processing volume $(R=0.495, p<0.05)$, and serving population $(R=0.591$, $p<0.05)$. Furthermore, the relationship between treatment techniques and the total concentration of OPEs in sewage sludge was also investigated in this study, and the results illustrated that the levels of OPEs in sludge were independent of the solid retention time (SRT).
\end{abstract}

๑) 2016 Elsevier Ltd. All rights reserved.

\footnotetext{
* Corresponding author.Department of Material and Chemical Engineering, Zhengzhou University of Light Industry, Zhengzhou, Henan 450001, People's Republic of China

** Corresponding author.

E-mail addresses: lpang@zzuli.edu.cn (L. Pang), zjh@zzuli.edu.cn (J. Zhao).
}

\section{Introduction}

In recent years, with the extensive use of flame retardants and plasticizers, organophosphate esters (OPEs) have regained recognitions and been regarded as a type of reemerging pollutants. While chlorinated OPEs such as tris(2-chloroethyl)phosphate (TCEP), tris(chloroisopropyl)phosphate (TCPP), and 
tris(dichloropropyl)phosphate (TDCP) are used as flame retardants, nonhalogenated OPEs such as tris(2-butoxyethyl)phosphate (TBEP), tributyl phosphate (TnBP), and triphenyl phosphate (TPhP) are mainly used as plasticizers, lubricants, antifoaming agents, and additives (Andresen et al., 2004). Because of the ban on the use of some polybrominated diphenyl ethers (PBDEs), mainly including penta-BDE, octa-BDE, and deca-BDE, in the European Union since 2008 (Betts, 2008), the use of OPEs as flame retardants has been increasing worldwide. Most OPEs have been regarded as highproduction volume chemicals, with volumes exceeding $1000 \mathrm{t}$ per year in Western Europe (Marklund et al., 2005; Stapleton et al., 2011).

As OPEs are not chemically bound to the appliances, these compounds can be released into the environment by volatilization, abrasion, and dissolution, and undergo long range atmospheric transportation (Reemtsma et al., 2008; Lai et al., 2015). Therefore, OPEs have been widely detected in the aquatic (Peverly et al., 2015; Zeng et al., 2014), terrestrial (Matsukami et al., 2015), and atmospheric environments (Mizouchi et al., 2015). There is an increased risk of exposure of OPEs to people, as previous studies have reported the presence of OPEs and their metabolites in human hair, nail (Liu et al., 2015; Cequier et al., 2015), and urine (Cequier et al., 2014). Extensive studies have already been conducted on the occurrence of OPEs, indicating that municipal wastewater from wastewater treatment plants (WWTPs) were the main contributors of OPEs to the aquatic environment (Fries and Puttmann, 2003, 2001). It was reported that the concentrations of OPEs were in the range of nanograms per liter to a few micrograms per liter in WWTP influents and effluents from different countries, such as South Korea (92-2620 ng L L ${ }^{-1}$ (Kim et al., 2007), Austria (20-900 ng $\mathrm{L}^{-1}$ ) (Martinez-Carballo et al., 2007), Spain (20-3700 ng L ${ }^{-1}$ ) (Rodil et al., 2009; Garcia-Lopez et al., 2008), Germany (25-62,000 ng L ${ }^{-1}$ ) (Rodil et al., 2005; Bester, 2005; Quintana et al., 2006), and Sweden (40-3000 ng L ${ }^{-1}$ ) (Marklund et al., 2005). Some OPEs, particularly hydrophobic OPEs, with low biodegradation and high bonding capacity to organic matters, are prone to be absorbed by organic compounds (Pang et al., 2013), which is considered as an important reason for the decrease of OPE concentrations in WWTP effluents (Bester, 2005; Marklund et al., 2005). Because of their high adsorption capacity onto sludge, the concentration of OPEs in sludge is relatively higher, in the range of micrograms per kilogram to a few milligrams per kilogram, such as in Sweden (2.2-4600 $\mathrm{ng} \mathrm{g}^{-1}$ ) (Marklund et al., 2005), Germany (1700-5100 $\mathrm{ng} \mathrm{g}^{-1}$ ) (Bester, 2005), Norway (3870-4810 $\mathrm{ng} \mathrm{g}^{-1}$ ) (Green et al., 2008), and South China (96.7-1312.9 $\mu \mathrm{g} \mathrm{kg}^{-1}$ ) (Zeng et al., 2014). The untreated sewage sludge directly discharged into the environment causes serious environmental problems. Thus, it is necessary to investigate the pollution due to OPEs in China. Henan province is located in Central China, which is the main grainproducing area, and the industrial sector has been developing fast in the recent years. This study aimed to investigate the occurrence and distribution of six common OPEs in the sewage sludge from 24 WWTPs in 18 cities of Henan province. It also assesses the potential factors affecting the levels of OPEs in sewage sludge in selected WWTPs. As the number of studies on pollution cause by OPEs in China is limited, this study helps to realize the pollution levels, occurrence, and distribution of OPEs, particularly in Central China.

\section{Material and methods}

\subsection{Chemicals and reagents}

Six organophosphates of flame retardant standard were purchased from Dr. Ehrenstorfer GmbH (Augsburg, Germany): TCEP, TCPP, TDCP, TnBP, TPhP, and TBEP. Surrogate tri- $n$-butyl- $\mathrm{d}_{27}$ phosphate (TnBP- $\mathrm{d}_{27}, 98 \%$ ) was purchased from Cambridge Isotope Laboratories (Andover, MA, USA). Acetonitrile (ACN), ethyl acetate, and methanol were obtained from Fisher Scientific (Shanghai, China), and all solvents used in this study were of chromatographic grade. Ultrapure water (18.2 M $\Omega$ ) was provided by Milli-Q Gradient system (Millipore, Bedford, MA, USA) in our laboratory. Individual OPE stock solutions ( $1000 \mathrm{mg} \mathrm{L}^{-1}$ ) were prepared in $\mathrm{ACN}$. The mixed stock solution containing $10 \mathrm{mg} \mathrm{L}^{-1}$ each of the six OPEs was prepared by diluting the stock solutions with water/ACN (50/50, v/ v). All the stock solutions were stored in the dark at $4^{\circ} \mathrm{C}$.

\subsection{Sample collection}

The sampling map and locations are shown in Fig. 1. A total of 24 sewage sludge samples were collected from different WWTPs in 18 cities in Henan province during the period of January to March 2015. Treatment technique, sewage sources, treatment capacity, serving population, and total organic carbon (TOC) values of sludge samples in each WWTP are summarized in Table S1.

The sludge samples collected in this study were dewatered ones, which were stored in glass bottles. They were stored at $-20^{\circ} \mathrm{C}$ until analysis. The samples were then freeze-dried and ground fine. After sieved through 100 mesh, the sludge samples were stored in amber glass bottles until extraction. Glasswares were used in this study instead of plastic containers to avoid the interference of OPEs.

\subsection{Sample pretreatment}

The target OPEs were extracted using ultrasonic assistance according to the published procedures with minor modifications (Cao et al., 2012). In brief, $0.5 \mathrm{~g}$ of dry sludge from each sample was spiked with $10 \mu \mathrm{L}$ of $\mathrm{TnBP}_{-} \mathrm{d}_{27}$ at $5 \mathrm{mg} \mathrm{L}^{-1}$ as surrogate and then extracted by ultrasonic assistance with $20 \mathrm{~mL}$ of ACN/water (25/75, $\mathrm{v} / \mathrm{v}$ ) at ambient temperature for $30 \mathrm{~min}$. The extract was centrifuged at $9000 \mathrm{rpm}$ and $20^{\circ} \mathrm{C}$ for $15 \mathrm{~min}$ by using polytetrafluoroethylene (PTEF) centrifuge tube, and the supernatant was decanted into a $500 \mathrm{~mL}$ volumetric flask. This process was performed thrice and the combined extract was diluted to $500 \mathrm{~mL}$ by ultrapure water. Oasis HLB extraction cartridges ( $200 \mathrm{mg}, 6 \mathrm{~mL}$ ) were used for further separation and concentration. Before loading the water samples, the solid-phase extraction (SPE) cartridges were preconditioned with $4 \mathrm{~mL}$ of ACN and water sequentially. After loading, the cartridges were washed with $3 \mathrm{~mL}$ of water and dried with air for $10 \mathrm{~min}$. The enriched analytes were eluted twice with $8 \mathrm{~mL}$ of ACN. After being concentrated to almost dryness with a gentle flow of $\mathrm{N}_{2}$ using a nitrogen evaporator (Anpel, Shanghai, China), the resulting extract was subsequently diluted to approximately $500 \mu \mathrm{L}$ with ACN/water $(50 / 50, v / v)$, and an aliquot $(10 \mu \mathrm{L})$ of the solution was injected into the high-performance liquid chromatographyelectrospray ionization (HPLC-ESI) MS/MS (ACQUITY UPLC, Waters, USA) system for analysis. The detection parameters of the samples under study are shown in Table S2.

TOC was measured as the $\mathrm{CO}_{2}$ content on acid-treated samples using a solid TOC analyzer (Shimadzu SSM-5000A, Japan). TOC concentration was calculated by the summation of organic and inorganic carbons.

\subsection{Quantitation and quality control}

Field blanks $(n=4)$, procedural blanks $(n=4)$, spiked blanks $(n=4)$, spiked matrix $(n=4)$, and replicate samples $(n=7)$ were analyzed with extraction to control contamination. In each spiked sample, 50- and 100-ng mixtures of six OPEs were added. All samples were spiked with TnBP-d27 as surrogate. TBEP and TnBP were not found in the blank; TPhP was found at the limited of 




Fig. 1. Sampling locations of municipal sewage sludge samples in Henan province, Central China.

detection (LOD); and TCEP, TDCP and TCPP were detected at 24.8, 24.7 and $12.4 \mathrm{ng} \mathrm{L}^{-1}$ in blanks. The recoveries of the selected OPEs were evaluated by adding of 50- and 100-ng mixtures of standards containing six OPEs into the mixture of three sludge samples. Table S3 shows acceptable recoveries of target OPEs in the range of $53.2-127 \%$ and $52.9-127 \%$, respectively. Furthermore, the matrix effect was also evaluated by adding 50- and 100-ng mixtures of standard solutions into the pre-extracted sludge, and appreciable recoveries were achieved in the range of $98.3-121 \%$ and $91.5-128 \%$. The calibration curve ranged from 0.10 to $300 \mu \mathrm{g} \mathrm{L}^{-1}$, and was found to be highly linear $\left(r^{2}>0.994\right)$. The LODs and recoveries of OPEs from the sludge are listed in Table S3.

\section{Results and discussion}

\subsection{Levels of OPEs in sewage sludge}

Concentrations and detection rates of six OPEs in 24 sludge samples are listed in Table 1. The concentration of individual OPEs illustrated by box-and-whisker plots in Figure S1. The results showed that most of the studied OPEs were detected in all sludge samples and the average concentration of each OPE ranged from 15.1 to $43.3 \mu \mathrm{g} \mathrm{kg}^{-1}$. While, it is evident that TCEP, TBEP, and TCPP were predominant OPEs in sewage. Sludge with mean concentrations of $43.3,36.0$, and $33.6 \mu \mathrm{g} \mathrm{kg}^{-1}$, respectively. It is evident from Table 1 that the total concentration of OPEs ranged from 38.6 to $508 \mu \mathrm{g} \mathrm{kg}^{-1}$ (mean $170 \mu \mathrm{g} \mathrm{kg}{ }^{-1}$, dw). High concentrations of OPEs detected in the sludge were from the WWTPs located in Zhengzhou (mean $358 \mu \mathrm{g} \mathrm{kg}^{-1}, \mathrm{dw}$ ), Xinxiang (mean $293 \mu \mathrm{g} \mathrm{kg}^{-1}, \mathrm{dw}$ ), and Luoyang (mean $271 \mu \mathrm{g} \mathrm{kg}{ }^{-1}, \mathrm{dw}$ ), whereas, low concentrations were detected in the WWTPs located in Xinyang $\left(38.6 \mu \mathrm{g} \mathrm{kg}^{-1}, \mathrm{dw}\right)$ and Jiyuan (mean $54.1 \mu \mathrm{g} \mathrm{kg}^{-1}$, dw).

As illustrated in Figure S2, the composition profiles of target OPEs collected from the studied WWTPs showed that TCEP, TBEP, and TCPP comprise the majority of OPEs in sewage sludge, accounting for approximately $60.1 \%$ of the total OPEs. While, the level of TPhP in sludge was relatively low, and only accounted for $10.9 \%$ of the total OPEs. Furthermore, sludge samples collected from different cities exhibited different OPE composition profiles, which might be because pollution caused by OPEs depends on the industrial development types and levels of city. On the contrary, antibiotics showed similar composition profiles in sewage sludge samples (Li et al., 2013).

The total concentrations of OPEs detected in the sewage sludge in this study were almost twice lower than those found in South China (96.7-1312.9 $\mu \mathrm{g} \mathrm{kg}^{-1}$ ) (Zeng et al., 2014). Furthermore, the OPE concentrations in sewage sludge were found to be at least seven times lower than that of Sweden $\left(2.2-4600 \mathrm{ng} \mathrm{g}^{-1}\right.$ ) (Marklund et al., 2005), Germany (1700-5100 ng g ${ }^{-1}$ ) (Bester, 2005), and Norway (3870-4810 $\mathrm{ng} \mathrm{g}^{-1}$ ) (Green et al., 2008). The results indicated that the levels of pollution caused by OPEs in Henan province were relatively low, whereas that in the south coastal areas was relatively higher than the central areas of China.

\subsection{Comparison of different WWTPS}

The concentrations of individual and total OPEs in different WWTPs are listed in Table 1 and Figure S3. The highest and lowest levels of total OPEs were found at ZZ-4 (508 $\mu \mathrm{g} \mathrm{kg} \mathrm{kg}^{-1}$, dw), Zhengzhou, and XY-1 (38.6 $\left.\mu \mathrm{g} \mathrm{kg}^{-1}, \mathrm{dw}\right)$, Xinyang, respectively. 
Table 1

Concentrations of targeted OPEs in 24 WWTPs ( $\mu \mathrm{g} \mathrm{kg}^{-1}$, dw).

\begin{tabular}{|c|c|c|c|c|c|c|c|c|c|}
\hline No. & Cities & WWTPs & TBEP & TCEP & TDCP & TnBP & TPhP & ТСРP & Total \\
\hline 1 & \multirow[t]{5}{*}{ Zhengzhou } & ZZ-1 & 383 & 5.00 & 2.00 & 5.80 & 9.00 & 19.2 & 424 \\
\hline 2 & & $\mathrm{ZZ}-2$ & 83.2 & 69.7 & 48.4 & 32.4 & 46.4 & 83.9 & 364 \\
\hline 3 & & ZZ-3 & 48.0 & 21.5 & 28.0 & 53.2 & 16.9 & 60.7 & 228 \\
\hline 4 & & $Z Z-4$ & 66.3 & 185 & 34.8 & 29.4 & 31.8 & 161 & 508 \\
\hline 5 & & ZZ-5 & 67.0 & 45.7 & 30.9 & 31.0 & 38.7 & 52.9 & 266 \\
\hline 6 & Zhongmu & ZM-1 & 24.3 & 2.50 & 39.2 & 12.4 & 9.90 & 42.6 & 131 \\
\hline 7 & Xinzheng & $X Z-1$ & 1.70 & 11.1 & 9.60 & 13.4 & 11.9 & 7.00 & 54.7 \\
\hline 8 & \multirow[t]{2}{*}{ Xinmi } & XM-1 & 7.80 & 21.0 & 8.10 & 23.4 & 13.0 & 65.7 & 139 \\
\hline 9 & & XM-2 & 5.60 & 37.7 & LOD & 16.5 & 4.40 & 33.5 & 97.7 \\
\hline 10 & \multirow[t]{2}{*}{ Luoyang } & LY-1 & 73.3 & 65.2 & 24.1 & 23.6 & 20.5 & 51.7 & 258 \\
\hline 11 & & LY-2 & 6.20 & 203 & 7.60 & 13.8 & 21.7 & 31.5 & 284 \\
\hline 12 & Zhumadian & ZZD-1 & 27.8 & 12.0 & 9.00 & 58.4 & 7.20 & 14.1 & 129 \\
\hline 13 & Xinxiang & $\mathrm{XX}-1$ & 9.50 & 31.4 & 17.0 & 197 & 12.8 & 25.3 & 293 \\
\hline 14 & Sanmenxia & SMX-1 & 1.60 & 17.0 & 18.1 & 17.8 & 20.1 & 12.5 & 87.1 \\
\hline 15 & Xinyang & $X Y-1$ & 5.40 & 7.00 & 6.80 & 7.40 & 5.00 & 7.00 & 38.6 \\
\hline 16 & Kaifeng & KF-1 & 5.90 & 12.9 & 14.2 & 10.2 & 8.70 & 6.70 & 58.6 \\
\hline 17 & Puyang & PY-1 & 6.90 & 9.50 & 19.5 & 12.6 & 17.5 & 22.3 & 88.3 \\
\hline 18 & Jiyuan & JY-1 & 11.1 & 12.3 & 5.80 & 3.50 & 6.10 & 15.3 & 54.1 \\
\hline 19 & Shangqiu & SQ-1 & 5.40 & 16.1 & 11.7 & 4.40 & 8.30 & 23.2 & 69.1 \\
\hline 20 & Luohe & LH-1 & 4.10 & 24.6 & 7.80 & 4.40 & 7.90 & 17.2 & 66.0 \\
\hline 21 & Zhoukou & ZK-1 & 11.1 & 12.5 & 43.5 & 12.1 & 6.00 & 10.3 & 95.5 \\
\hline 22 & Jiaozuo & $\mathrm{JZ}-1$ & 2.10 & 102 & 7.20 & 8.00 & 10.1 & 17.0 & 146 \\
\hline 23 & Nanyang & NY-1 & 4.20 & 10.9 & 8.80 & 11.5 & 12.9 & 12.0 & 60.3 \\
\hline 24 & Hebi & HB-1 & 3.20 & 103 & 8.20 & 7.00 & 14.6 & 13.5 & 150 \\
\hline \multicolumn{3}{|c|}{ Mean } & 36.0 & 43.3 & 17.8 & 25.4 & 15.1 & 33.6 & 170 \\
\hline \multicolumn{3}{|c|}{ Detection rates (\%) } & 100 & 100 & 95.8 & 100 & 100 & 100 & \\
\hline
\end{tabular}

Zhengzhou is the capital of Henan province, and Xinyang is an agricultural city with a relatively low level of industrial development. Potential factors affecting the levels of OPEs in sewage sludge, such as characteristics of wastewater (domestic, industrial, or combined) and sludge (TOC), and the design and operational conditions of WWTPs (eg., treatment technique, treatment capacity, processing volume, and serving population) were also investigated (Table S1).

\subsubsection{Composition of the sewage}

As an important group of flame retardants and plasticizers, OPEs are widely used in daily life and industrial production. Thus, domestic sewage and industrial wastewater, as the main source of WWTPs, have potential impact on the concentration of OPEs in sewage sludge. Among the 24 WWTPs investigated in this study, 10 were fed with both domestic sewage and industrial wastewater, 13 with domestic sewage, and one (XM-2) was fed with pulping wastewater. The average concentration of OPEs in the sludge samples fed with combined water (mean $160 \mu \mathrm{g} \mathrm{kg}^{-1}$ ) was lower than those fed with domestic sewage (mean $184 \mu \mathrm{g} \mathrm{kg}^{-1}$ ). Moreover, the concentration of OPEs in the waste sludge samples from paper mills was relatively low (XM-2, mean $97.7 \mu \mathrm{g} \mathrm{kg}^{-1}$, dw). OPEs can be released from plastic productions, plastic pipeline, and fire retardant paints, all of which are widely used in daily life and industrial production. Thus, both domestic sewage and industrial wastewater contain relatively high levels of OPE. However, OPEs are scarcely used in paper production, and small quantity of OPEs may be released from plastic industrial pipeline and the production of auxiliary materials (Rodil et al., 2009). As mentioned earlier, the influence of sewage source on the concentrations of OPEs in waste sludge was complex.

\subsubsection{Treatment capacity, processing volume, and serving population}

The main design and operating parameters of WWTPs, treatment capacity, processing volume, and serving population were investigated in this study to assess the influence of OPEs. It can be seen from Table 2 that the total concentration of OPEs has no significant relationship with the individual parameters $(p>0.05)$. Similar results were found in previous studies that the levels of short-chain chlorinated paraffins, benzotriazole ultraviolet (UV) stabilizers, and antibiotics were irrelevant with the WWTP characteristics (Ruan et al., 2012; Zeng et al., 2012; Li et al., 2013). However, significant correlations were found between TPhP and treatment capacity $(\mathrm{R}=0.481, p<0.05)$, processing volume $(\mathrm{R}=0.495, p<0.05)$ and serving population $(\mathrm{R}=0.564, p<0.05)$. The results showed that the concentration of TPhP in sludge increases with the increase of sewage quantity and serving population. With regard to some pollutants, although the total concentration has no significant relationship with the individual parameters, significant correlations were found between total concentrations and the ratio of some parameters, such as antibiotics. Significant correlations were also found between the total concentrations and the ratio of processing volume to serving population (Li et al., 2013). However, similar results were not found for OPEs in this study, possibly because of the complex sources of OPEs in the environment.

\subsubsection{TOC in the sewage sludge}

TOC is an important property of solid matrices in the environment, having a significant impact on the adsorption, migration, and transformation of target compounds. Therefore, in order to assess the potential influence of TOC on the OPE levels, the contents of TOC (\%) in sludge samples were investigated. As shown in Table S1, the TOC contents of 24 sludge samples varied from $7.23 \%$ to $33.1 \%$. The Pearson's coefficients of the concentrations of OPEs and TOC of the sludge are presented in Table 2. No significant correlation between TOC and total concentrations as well as individual concentration of OPEs was found ( $p>0.05$ ), indicating that OPEs in sludge were independent of the organic content of the sludge. This may be due to their weak binding capacity with organic matter in sewage sludge, because of the low Kow values of TBEP and TCEP. According to the results of previous studies, OPEs with relatively higher Kow values could have a higher adsorption capability to TOC (Pang et al., 2013). However, in this study, the concentration of all target OPEs did not exhibit a significant correlation with TOC, which should be 
Table 2

Pearson's correlation matrix for the concentration of target OPEs with parameters of 24 WWTPs and TOC in the sludge.

\begin{tabular}{|c|c|c|c|c|c|c|c|}
\hline Parameters & TBEP & TCEP & TDCP & TnBP & $\mathrm{TPhP}$ & TCPP & Total \\
\hline Treatment capacity $\left(10^{4} \mathrm{~m}^{3} /\right.$ day $)$ & $0.045(0.833)$ & $-0.078(0.716)$ & $0.340(0.104)$ & $0.188(0.379)$ & $0.481^{\mathrm{a}}(0.017)$ & $0.215(0.356)$ & $0.187(0.383)$ \\
\hline Processing volume $\left(10^{4} \mathrm{~m}^{3} /\right.$ day $)$ & $0.023(0.918)$ & $0.003(0.990)$ & $0.360(0.091)$ & $0.166(0.448)$ & $0.495^{\mathrm{a}}(0.016)$ & $0.312(0.147)$ & $0.228(0.296)$ \\
\hline Population $\left(10^{4}\right)$ & $-0.056(0.849)$ & $0.028(0.923)$ & $0.487(0.077)$ & $0.338(0.238)$ & $0.564^{\mathrm{a}}(0.036)$ & $0.428(0.127)$ & $0.300(0.298)$ \\
\hline Ratio 1 & $-0.157(0.592)$ & $0.490(0.076)$ & $-0.153(0602)$ & $-0.052(0860)$ & $0.003(0.992)$ & $0.155(0.597)$ & $0.014(0.962)$ \\
\hline Ratio 2 & $-0.165(0.573)$ & $-0.037(0.900)$ & $0.319(0.266)$ & $0.074(0.801)$ & $0.208(0.476)$ & $0.488(0.077)$ & $0.043(0.883)$ \\
\hline TOC (\%) & $-0.373(0.095)$ & $-0.114(0.622)$ & $0.228(0.321)$ & $-0.044(0.849)$ & $0.225(0.327)$ & $-0.206(0.371)$ & $-0.291(0.200)$ \\
\hline
\end{tabular}

a Correlation is significant at the 0.05 level (two-tailed).

investigated in further studies.

\subsubsection{Treatment technique}

Principal component analysis (PCA) was performed to assess the characteristics of OPEs in sewage sludge of WWTPs under study. The detectable rates of all target OPEs were $>65 \%$ and the average concentration was $>1.00 \mu \mathrm{g} \mathrm{kg}^{-1}$. The varimax rotation reduced all variables into three principal components (PCs) with eigenvalues $>1$, which represent $76.9 \%$ of the cumulative variances. The loading and score plots of the three PCs are shown in Fig. 2. It can be
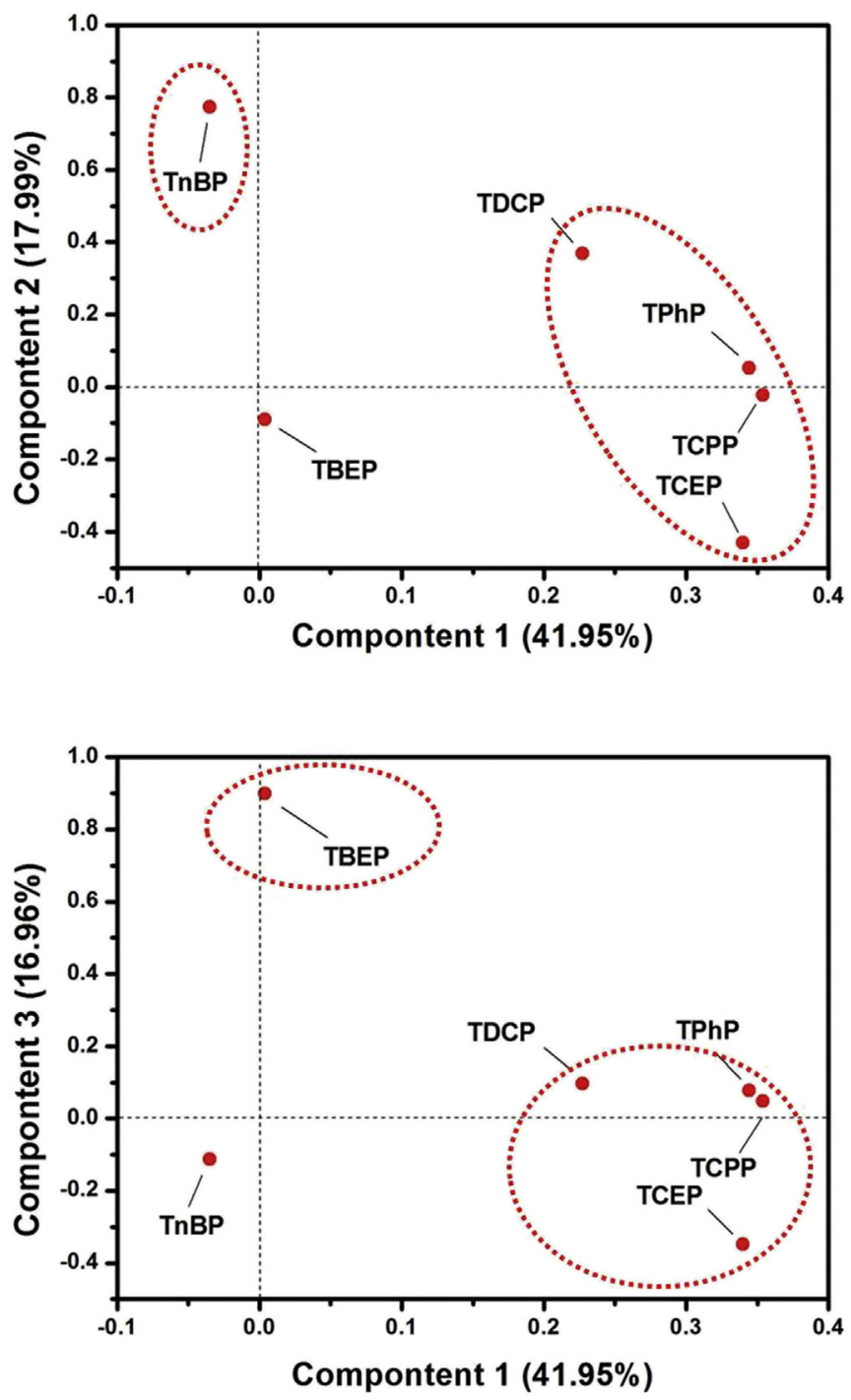

Fig. 2. The Loading plots of PCA by the database of OPEs in sewage sludge in Henan Province, Central China. observed that PC1 accounted for $41.95 \%$ of the total variance and had moderately positive loadings on TCPP, TPhP, TCEP, and TDCP;


Fig. 3. Score plots of PCA by the database of OPEs with different treatment techniques from 24 WWTPs. (a) PC1 vs. PC2; (b) PC1 vs. PC3. 
PC2 accounted for $17.99 \%$ of the total variance and had high positive loading for TnBP; and PC3 accounted for $16.96 \%$ of the total variance, and was highly correlated with TBEP.

The score plots of PCA in Fig. 3 were grouped by treatment techniques to visualize potential relationships. PCA results showed that WWTPs in the University of Cape Town (UCT) and anoxic/oxic (AO) have higher loadings of PC1 and PC2 in the component plot, indicating higher concentrations of TCPP, TPhP, TCEP, TDCP, and TnBP in these WWTPs. Anaerobic/anoxic/oxic (AAO) has higher loadings of PC3, indicating the higher level of TBEP in AAO WWTPs. By contrast, the other two treatment techniques, cyclic activated sludge system (CASS) and oxidation ditch (OD), showed low loading values of two PCs. This result indicated that low levels of these OPEs were found in these types of WWTPs.

Because the wastewater treatment techniques of WWTPs have potential impact on the concentrations of some organic pollutants in influents and effluents (Yu and Chu, 2009; Li et al., 2013), the relationship between treatment techniques and the total concentrations of OPEs in sewage sludge was also investigated. Main treatment techniques of the investigated WWTPs are listed in Table S1, including AO, AAO, OD, UCT, and CASS activated sludge processes. As shown in Figure S4, the average total concentrations of OPEs treated by the aforementioned activated sludge processes in the sludge samples were in the order of $\mathrm{AO}\left(198 \mu \mathrm{g} \mathrm{kg}^{-1}\right.$, $\mathrm{dw})>$ AAO $\left(171 \mu \mathrm{g} \mathrm{kg}^{-1}, \mathrm{dw}\right)>$ UCT $\left(169 \mu \mathrm{g} \mathrm{kg}^{-1}, \mathrm{dw}\right)>$ OD $\left(150 \mu \mathrm{g} \mathrm{kg}^{-1}, \mathrm{dw}\right)>$ CASS $\left(54.7 \mu \mathrm{g} \mathrm{kg}^{-1}, \mathrm{dw}\right)$. As the one of the important operating parameters, solid retention time (SRT) varied with treatment technique. Therefore, SRT may have a potential impact on the concentration of OPEs in sludge (Batt et al., 2007; Li et al., 2013.). In general, OD and CASS possess a relatively long SRT (20-40 days), AAO and UCT operate with an SRT of 10-20 days, and AO possesses the shortest SRT (3-10 days) for relatively simple processes. Thus, it is generally believed that the concentrations of target compounds in sewage sludge would be high with longer SRTs. However, in this study, there was no significant relationship between the concentration of OPEs in sludge and SRT. The possible reasons were the concentrations of OPEs in sewage influent influence the OPE levels in sludge; besides, the release of OPEs from plastic pipes during the treatment procedure was an important source for their concentration in sewage sludge (Rodil et al., 2009).

Therefore, it can be proved that the relatively high concentrations of OPEs can be found in sewage sludge. Hence, the decrease of OPEs in sewage effluent was completely dependent on not only the degradation processes during sewage treatment, but also the removal by sorption on the sludge (Hyland et al., 2012). However, the major reasons for the decrease of OPEs in WWTPs effluents were not investigated in this study, and further research is necessary.

\section{Conclusions}

In this study, as a type of reemerging pollutants, six common OPEs were detected in sewage samples, which were collected from 24 WWTPs of 18 cities in Henan province, Central China. The detection rates of all selected OPEs were $>90 \%$, illustrating the universal usage and extensive pollution in Henan province. TCEP, TBEP, and TCPP were the predominant OPEs found in sludge samples. High concentrations of OPEs were found in the sludge from WWTPs located in Zhengzhou, Xinxiang, and Luoyang, whereas low concentrations were found in those located in Xinyang and Jiyuan. The level of TPhP in sewage sludge was associated with the treatment capacity, processing volume, and serving population, but similar results were not found for other OPEs. However, there was no significant relationship between the total concentration of OPEs and treatment techniques, which might be due to the complex influence on the concentrations of OPEs, and further investigations are necessary. Thus, it could be concluded that from this study that the sorption of OPEs to sludge is one of the main reasons for the decrease in the concentration of OPEs in WWTP effluents.

\section{Acknowledgments}

This study was supported by the Open foundation Major Science and Technology Program for Water Pollution Control and Treatment (2015ZX07204-002-003), State Key Laboratory of Environmental Chemistry and Ecotoxicology (KF2013-13), and Zhengzhou Committee of Science and Technology, China (141PPTGG412).

\section{Appendix A. Supplementary data}

Supplementary data related to this article can be found at http:// dx.doi.org/10.1016/j.chemosphere.2016.02.104.

\section{References}

Andresen, J.A., Grundmann, A., Bester, K., 2004. Organophosphorus flame retardants and plasticisers in surface waters. Sci. Total. Environ. 332, 155-166.

Batt, A.L., Kim, S., Aga, D.S., 2007. Comparison of the occurrence of antibiotics in four full-scale wastewater treatment plants with varying designs and operations. Chemosphere 68, 428-435.

Bester, K., 2005. Comparison of TCPP concentrations in sludge and wastewater in a typical German sewage treatment plant-comparison of sewage sludge from 20 plants. Environ. Monit. 7, 509-513.

Betts, K., 2008. Does a key PBDE break down in the environment? Environ. Sci. Technol. 42, 6781-6781.

Cao, S.X., Zeng, X.Y., Song, H., Li, H.R., Yu, Z.Q., Sheng, G.Y., Fu, J.M., 2012. Levels and distributions of organophosphate flame retardants and plasticizers in sediment from taihu lake, China. Environ. Toxicol. Chem. 31, 1478-1484.

Cequier, E., Sakhi, A.K., Marce, R.M., Becher, G., Thomsen, C., 2015. Human exposure pathways to organophosphate trimesters-A biomonitoring study of mother-child pairs. Environ. Int. 75, 159-165.

Cequier, E., Marce, R.M., Becher, G., Thomsen, C., 2014. A high-throughput method for determination of metabolites of organophosphate flame retardants in urine by ultra performance liquid chromatography-high resolution mass spectrometry. Anal. Chim. Acta 845, 98-104.

Fries, E., Puttmann, W., 2003. Monitoring of the three organophosphate esters TBP, TCEP and TBEP in river water and ground water (Oder, Germany). J. Environ. Monit. 5, 346-352.

Fries, E., Puttmann, W., 2001. Occurrence of organophosphate esters in surface water and ground water in Germany. J. Environ. Monit. 3, 621-626.

Garcia-Lopez, M., Rodriguez, I., Cela, R., 2008. Evaluation of liquid-liquid microextraction using polypropylene microporous membranes for the determination of organophosphorus flame retardants and plasticizers in water samples. Anal. Chim. Acta 625, 145-153.

Green, N., Schlabach, M., Bakke, T., Brevik, E.M., Dye, C., Herzke, D., Huber, S., Plosz, B., Remberger, M., Schoyen, M., Uggerud, H.T., Vogelsang, C., 2008. In: Screening of Selected Metals and New Organic Contaminants 2007. Norwegian Pollution Control Agency.

Hyland, K.C., Dickenson, E.R., Drewes, J.E., Higgins, C.P., 2012. Sorption of ionized and neutral emerging trace organic compounds onto activated sludge from different wastewater treatment configurations. Water. Res. 46, 1958-1968.

Kim, S.D., Chao, J., Kim, I.S., Vanderford, B.J., Snyder, S.A., 2007. Occurrence and removal of pharmaceuticals and endocrine disruptors in South Korean surface, drinking, and waste waters. Water. Res. 41, 1013-1021.

Lai, S.C., Xie, Z.Y., Song, T.L., Tang, J.H., Zhang, Y.Y., Mi, W.Y., Peng, J.H., Zhao, Y., Zou, S.C., Ebinghaus, R., 2015. Occurrence and dry deposition of organophosphate esters in atmospheric particles over the northern South China Sea. Chemosphere 127, 195-200.

Li, W.H., Shi, Y.L., Gao, L.H., Liu, J.M., Cai, Y.Q., 2013. Occurrence, distribution and potential affecting factors of antibiotics in sewage sludge of wastewater treatment plants in China. Sci. Total. Environ. 445-446, 306-313.

Liu, L.Y., Salamova, A., He, K., Hites, R.A., 2015. Analysis of polybrominated diphenyl ethers and emerginghalogenated and organophosphate flame retardants in human hairand nails. J. Chromatogr. A 1406, 251-257.

Marklund, A., Andersson, B., Haglund, P., 2005. Organophosphorus flame retardants and plasticizers in Swedish sewage treatment plants. Environ. Sci. Technol. 39, $7423-7429$.

Martinez-Carballo, E., Gonzalez-Barreiro, C., Sitka, A., Scharf, S., Gans, O., 2007. Determination of selected organophosphate esters in the aquatic environment of Austria. Sci. Total. Environ. 388, 290-299.

Matsukami, H., Tue, N.M., Suzuki, G., Someya, M., Tuyen, L.H., Viet, P.H., Takahashi, S., Tanabe, S., Takigami, H., 2015. Flame retardant emission from ewaste recycling operation in northern Vietnam: environmental occurrence of emerging organophosphorus esters used as alternatives for PBDEs. Sci. Total. 
Environ. 514, 492-499

Mizouchi, S., Ichiba, M., Takigani, H., Kajiwara, N., Takamuku, T., Miyajima, T. Kodama, H., Someya, T., Ueno, D., 2015. Exposure assessment of organophosphorus and organobromine flame retardants via indoor dust from elementary schools and domestic houses. Chemosphere 123, 17-25.

Pang, L., Liu, J.F., Yin, Y.G., Shen, M.H., 2013. Evaluating the sorption of organophosphate esters to different sourced humic acids and its effects on the toxicity to Daphnia magna. Environ. Toxicol. Chem. 32, 2755-2761.

Peverly, A.A., O'Sullivan, C., Liu, L.Y., Venier, M., Martinez, A., Hornbuckle, K.C., Hites, R.A., 2015. Chicago's Sanitary and Ship Canal sediment: polycyclic aromatic hydrocarbons, polychlorinated biphenyls, brominated flame retardants, and organophosphate esters. Chemosphere 134, 380-386.

Quintana, J.B., Rodil, R., Reemtsma, T., 2006. Determination of phosphoric acid mono- and diesters in municipal wastewater by solid-phase extraction and ionpair liquid chromatography - tandem mass spectrometry. Anal. Chem. 78 1644-1650.

Reemtsma, T., Quintana, J.B., Rodil, R., Garcia-Lopez, M., Rodriguez, I., 2008. Organophosphorus flame retardants and plasticizers in water and air I. Occurrence and fate. TrAC. Trends Anal. Chem. 27, 727-737.

Rodil, R., Quintana, J.B., Lopez-Mahia, P., Muniategui-Lorenzo, S., PradaRodriguez, D.J., 2009. Multi-residue analytical method for the determination of emerging pollutants in water by solid-phase extraction and liquid chromatography-tandem mass spectrometry. J. Chromatogr. A 1216, 2958-2969.

Rodil, R., Quintana, J.B., Reemtsma, T., 2005. Liquid chromatography-tandem mass spectrometry determination of nonionic organophosphorus flame retardants and plasticizers in wastewater samples. Anal. Chem. 77, 3083-3089.

Ruan, T., Liu, R.Z., Fu, Q., Wang, T., Wang, Y.W., Song, S.J., Wang, P., Teng, M. Jiang, G.B., 2012. Concentrations and composition profiles of benzotriazole UV stabilizers in municipal sewage sludge in China. Environ. Sci. Technol. 46, 2071-2079.

Stapleton, H.M., Klosterhaus, S., Keller, A., Ferguson, P.L., van Bergen, S., Cooper, E., Webster, T.F., Blum, A., 2011. Identification of flame retardants in polyurethane foam collected from baby products. Environ. Sci. Technol. 45, 5323-5331.

Yu, C., Chu, K., 2009. Occurrence of pharmaceuticals and personal care products along the West Prong Little Pigeon River in east Tennessee, Usa. Chemosphere $75,1281-1286$.

Zeng, X.Y., He, L.X., Cao, S.X., Ma, S.T., Yu, Z.Q., Gui, H.Y., Sheng, G.Y., Fu, J.M., 2014 Occurrence and distribution of organophosphate flame retardants/plasticizers in wastewater treatment plant sludges from the Pearl River delta, China. Environ. Toxicol. Chem. 33, 1720-1725.

Zeng, L.X., Wang, T., Ruan, T., Liu, Q., Wang, Y.W., Jiang, G.B., 2012. Levels and distribution patterns of short chain chlorinated paraffins in sewage sludge of wastewater treatment plants in China. Environ. Pollut. 160, 88-94. 